\title{
Dynamic characteristics of a non-symmetric tilting pad journal bearing
}

\author{
Dang Phuoc Vinh ${ }^{10000-0002-9515-5173]}$, Steven Chatterton ${ }^{20000-0003-4170-009 X]}$ and Paolo \\ Pennacchi ${ }^{2[0000-0001-8174-0462] ~}$ \\ ${ }^{1}$ Dept. of Mechanical Engineering, The University of Danang - Danang University of Science \\ and Technology \\ ${ }^{2}$ Dept. of Mechanical Engineering, Politecnico di Milano, Via G. La Masa 1, 20145 Milan, \\ Italy \\ dpvinh@dut.udn.vn, \{steven.chatterton, paolo.pennacchi\}@polimi.it
}

\begin{abstract}
Because tilting-pad journal bearings are more stable and efficient than conventional bearings, they have been commonly applied to many rotating machinery applications. Most of the studies about steady state and dynamic characteristics of tilting-pad journal bearings are usually evaluated by means of thermo hydrodynamic models assuming nominal dimensions for the bearing. However machining errors could lead to actual bearing geometry and dimensions different from the nominal ones. In particular for tilting-pad journal bearing the asymmetry of the bearing geometry is the principal cause of unexpected behavior. In this paper a theoretical analysis on dynamic characteristics of a five-pad tilting-pad journal bearing is investigated with non-nominal geometry, that is, different thickness for each pad. The dynamic coefficients of a five-pad tilting-pad journal bearing with a nominal diameter of $100 \mathrm{~mm}$, length-todiameter ratio (L/D) of 0.7 are evaluated versus rotor rotational speed, load direction and static load. Then, the analytical results of the non-nominal bearing are compared to those of a bearing having nominal (i.e. ideal) geometry.

Keywords: Tilting-pad journal bearing, analytical model, non-nominal bearing, dynamic coefficients, five pads.
\end{abstract}

\section{Introduction}

Because tilting-pad journal bearings (TPJBs) are more stable and efficient than conventional bearings, they have been commonly applied to many rotating machinery applications. The chief feature of tilting-pads is that they modify their configuration to adapt to every operating condition, creating several convergent-divergent gaps around the circumference and thus making the system highly stable. Since Lund [1] developed a numerical method for calculating dynamic coefficients for tilting-pad journal bearings, extensive theoretical and experimental studies on dynamic and stability analysis have been conducted. In the course of the development of journal bearings, many effective methods have been applied, such as Newton-Raphson method, pad assembly technique, finite elements method, and Genetic Algorithm to calculate static as well as dynamic characteristics of journal bearing [2] - [3].

The majority of the papers on TPJB in the literature consider the tilting pad bearings with nominal dimensions [4]-[10]. Jones et al [11] studied theoretical the effects 
of bearing clearance and pad clearance on the steady-state and dynamic behavior in tilting pad journal bearings. They concluded that the pad clearance has little effect on the $K_{y y}$ term at small values of bearing clearance ratio and the bearing clearance has more effect than pad clearance.

Strzelecki [12] has studied the dynamic characteristics of tilting-pad bearings with asymmetric pad support using Reynolds equation and an adiabatic model for the oil film. It was applied to a five pad bearing, and then the pad relative clearance has influenced the stiffness and damping coefficients, while the length to the diameter ratio has just affected the direct stiffness coefficients.

However, most papers studied steady-state and dynamic characteristics of tiltingpad journal bearing by means of thermo hydrodynamic (THD) models assuming nominal dimensions for the bearing. It means that the physical dimensions of all pads are identical or the bearing is uniform configuration. In this paper, effects of non-uniform clearance on TPJBs' performances, namely dynamic coefficients and shaft locus are evaluated using an analytical model. A five-pad tilting-pad journal bearing with different thickness in one pad is analytically modeled. Its steady state and dynamic behaviors are compared with a symmetric tilting-pad journal bearing.

\section{Bearing description}

The sketch of the five-pads TPJB considered in this paper is shown in Fig. 1. The bearing is installed in its housing as a standard LOP configuration, with nominal diameter of $100 \mathrm{~mm}$ and length-to-diameter ratio of 0.7 . The geometric characteristics of the bearing and the operating conditions are listed in Table 1. $O_{b}$ and $O_{j}$ which are located at the origin of the $X-Y$ coordinate system denote for the center of the bearing and journal, respectively.

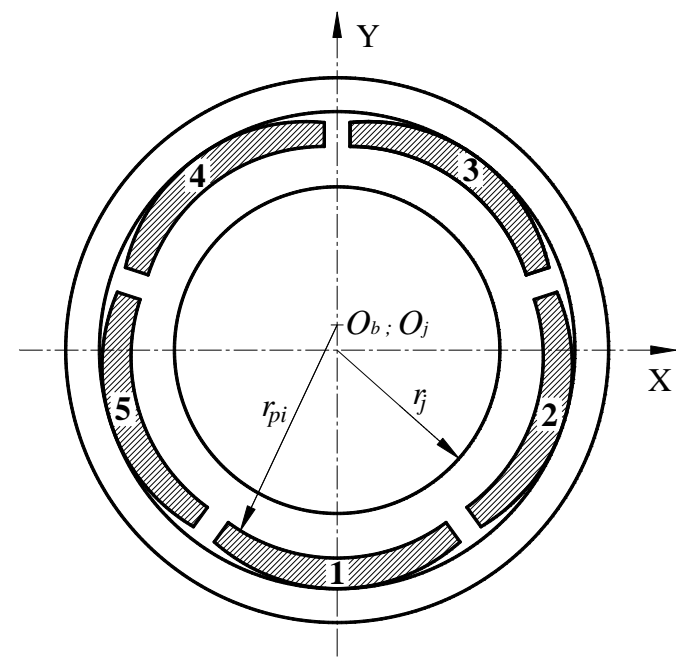

Fig. 1. Sketch of the five-pads TPJB

The main advantage of TPJB consists in the pads capability to follow the displacements of the rotor. During operation, each pad rotates so that the resultant of the fluid-film forces passes through the pivot. Thus, the pivot location influences the pad rotation and the magnitude of the hydrodynamic pressure distribution.

In this paper, a non-ideal TPJB, in which the thicknesses of the five pads are different from each other due to machining errors, is considered. The effect is a different assembled clearance or preload factor $\left(m=1-C_{b} / C_{p}\right)$ for each pad (see in Table 2). 
Table 1. Bearing geometric characteristics and operating conditions.

\begin{tabular}{lll}
\hline Item & Unit & Value/Span \\
\hline Number of pad & - & 5 \\
Configuration w.r.t bearing housing & - & LOP \\
Bearing diameter $(D)$ & $\mathrm{mm}$ & 100 \\
Machined clearance $\left(C_{p}\right)$ & $\mathrm{mm}$ & 0.125 \\
Bearing length $(L)$ & $\mathrm{mm}$ & 70 \\
Angular amplitude of pads & Degree $\left(^{\circ}\right)$ & 60 \\
Lubricant & - & ISO VG46 \\
Oil inlet temperature & ${ }^{\circ} \mathrm{C}$ & $38-40$ \\
Rotational speed & $\mathrm{rpm}$ & 1200 \\
Static load (on each bearing) & $\mathrm{kN}$ & 5 \\
\hline
\end{tabular}

Table 2. Specifications of the real tilting-pad journal bearing.

\begin{tabular}{|c|c|c|c|c|c|c|}
\hline \multirow{2}{*}{ Item } & \multicolumn{5}{|c|}{ Measurement } & Nominal \\
\cline { 2 - 6 } & Pad \#1 & Pad \#2 & Pad \#3 & Pad \#4 & Pad \#5 & dimension \\
\hline Thickness $(\mathrm{mm})$ & 15.9942 & 16.0146 & 15.9995 & 15.9812 & 16.0179 & 16.0 \\
\hline $\begin{array}{c}\text { Assembled clearance } \\
C_{b}(\mathrm{~mm})\end{array}$ & 0.0658 & 0.0454 & 0.0605 & 0.0788 & 0.0421 & 0.07 \\
\hline Preload factor $m$ & 0.4739 & 0.6365 & 0.5157 & 0.3698 & 0.6630 & 0.44 \\
\hline
\end{tabular}

\section{TEHD bearing model}

A thorough description of the TEHD model to estimate the static and dynamic behaviors of a five-pad TPJB is provided in [5]-[8].

The hydrodynamic model is based on the well-known Reynolds equation:

$$
\begin{aligned}
& \frac{\partial}{\partial x}\left(\frac{\rho h^{3}}{\mu} \frac{\partial p}{\partial x}\right)+\frac{\partial}{\partial z}\left(\frac{\rho h^{3}}{\mu} \frac{\partial p}{\partial z}\right) \\
& =6\left[\left(U_{1}-U_{2}\right) \frac{\partial h}{\partial x}+h \frac{\partial}{\partial x}\left(U_{1}+U_{2}\right)\right. \\
& \left.+\left(W_{1}-W_{2}\right) \frac{\partial h}{\partial z}+h \frac{\partial}{\partial z}\left(W_{1}+W_{2}\right)+2\left(V_{1}+V_{2}\right)\right]
\end{aligned}
$$

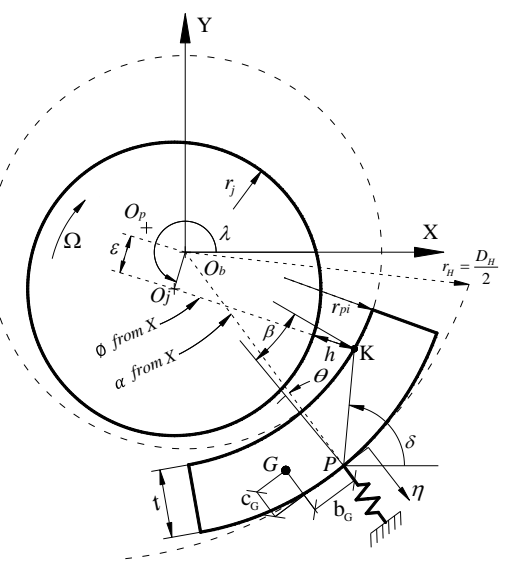

Fig. 2. Sketch of a rocker-backed TPJB [5].

where $p$ is the pressure in the oil-film, $h$ is the oil-film thickness, $\mu$ is the dynamic viscosity, $\rho$ is the density of oil, $z$ is the axial direction, $x$ is the tangential direction. The velocity vector component of the shaft and the pads are defined by $U_{1}, V_{1}, W_{1}$ and $U_{2}, V_{2}, W_{2}$, respectively. 
The effect of temperature on the dynamic viscosity $\mu(T)=\mu_{40^{\circ} \mathrm{C}} \exp \left[\kappa\left(T_{40^{\circ} \mathrm{C}}-T\right)\right]$ and oil density $\rho(T)=\rho_{40^{\circ} \mathrm{C}}\left[1+\alpha_{v}\left(T_{40^{\circ} \mathrm{C}}-T\right)\right]$ is considered using a simple twodimensional thermal model, governed, at steady state, by the energy equation:

$$
\rho c_{p}\left(u \frac{\partial T}{\partial x}+w \frac{\partial T}{\partial z}\right)=k_{\text {OIL }}\left(\frac{\partial^{2} T}{\partial x^{2}}+\frac{\partial^{2} T}{\partial z^{2}}\right)+\mu\left[\left(\frac{\partial u}{\partial y}\right)^{2}+\left(\frac{\partial w}{\partial y}\right)^{2}\right]
$$

where $\kappa$ is the viscosity index and $\alpha_{v}$ is the thermal expansion coefficient of the oil, $c_{p}$ and $k_{L}$ are the heat capacity and the conductivity of the lubricant respectively. Equation (2) has been integrated using a finite difference method, where adiabatic conditions at the shaft, pad surfaces and constant oil temperature in the oil film thickness direction are considered.

The dynamic coefficients of the bearing because of the lateral motion of the shaft (represented by four impedance coefficients of the impedance matrix $\left[\mathbf{Z}_{B R G}\right]$ in Eq. (3), are obtained in Eq. (4) by reducing the complete set of impedance coefficients of the pads (impedance matrix $[\mathbf{Z}]^{k}$ in Eq. (5), assuming a harmonic motion of the system at frequency $\omega[5]$ :

$$
\begin{gathered}
{\left[\begin{array}{l}
\Delta F_{x, o i l} \\
\Delta F_{y, o i l}
\end{array}\right]=-\left[\mathbf{Z}_{B R G}\right]\left[\begin{array}{l}
\Delta X \\
\Delta Y
\end{array}\right]=-\left[\begin{array}{ll}
Z_{x x} & Z_{x y} \\
Z_{y x} & Z_{y y}
\end{array}\right]\left[\begin{array}{c}
\Delta X \\
\Delta Y
\end{array}\right]} \\
{\left[\mathbf{Z}_{B R G}\right]=-\sum_{k}\left(\left[\begin{array}{ll}
Z_{x x} & Z_{x y} \\
Z_{y x} & Z_{y y}
\end{array}\right]^{k}-\left[\begin{array}{ll}
Z_{x \theta} & Z_{x \eta} \\
Z_{y \theta} & Z_{y \eta}
\end{array}\right]^{k}\left(\left[\begin{array}{ll}
Z_{\theta \theta} & Z_{\theta \eta} \\
Z_{\eta \theta} & Z_{\eta \eta}
\end{array}\right]^{k}-\omega^{2}\left[M_{p a d}\right]^{k}+\mathrm{i} \omega\left[C_{p a d}\right]^{k}+\left[K_{p a d}\right]^{k}\right)^{-1}\left[\begin{array}{ll}
Z_{\theta x} & Z_{\theta y} \\
Z_{\eta x} & Z_{\eta y}
\end{array}\right]^{k}\right)} \\
{[\mathbf{Z}]^{k}=[\mathbf{K}]^{k}+\mathrm{i} \omega[\mathbf{C}]^{k}=\left[\begin{array}{llll}
z_{x x} & z_{x y} & z_{x \theta} & z_{x \eta} \\
z_{y x} & z_{y y} & z_{y \theta} & z_{y \eta} \\
z_{\theta x} & z_{\theta y} & z_{\theta \theta} & z_{\theta \eta} \\
z_{\eta x} & z_{\eta y} & z_{\eta \theta} & z_{\eta \eta}
\end{array}\right]^{k}}
\end{gathered}
$$

where $[\mathbf{K}]^{k}$ and $[\mathbf{C}]^{k}$ are the linear stiffness and damping coefficient matrices, respectively, which are calculated for the $k$-th pad, and:

$$
\left[M_{p a d}\right]^{k}=\left[\begin{array}{cc}
J_{P} & m b_{G} \\
m b_{G} & m
\end{array}\right]^{k} \quad\left[C_{p a d}\right]^{k}=\left[\begin{array}{cc}
c_{\theta} & 0 \\
0 & c_{\eta}
\end{array}\right]^{k} \quad\left[K_{p a d}\right]^{k}=\left[\begin{array}{cc}
k_{\theta} & 0 \\
0 & k_{\eta}
\end{array}\right]^{k}
$$

where $J_{p}$ is the mass moment of inertia of the pad, $m$ is the mass, and $b_{G}$ is the position of the barycenter. The pivot stiffness of the pivot along the direction $\eta$, which depends on the applied static load $W$, is obtained using the contact Hertz theory.

\section{$4 \quad$ Results and discussion}

\subsection{Dynamic coefficients versus rotor rotational speed}

Fig. 3 shows the stiffness coefficients of two bearings as a function of rotational speed in LOP configuration when a static load of $5 \mathrm{kN}$ is applied on the bearing in the vertical direction. The direct stiffness coefficients $k_{x x}$ and $k_{y y}$ increase more or less 
linearly with increasing of rotational speed from $1000 \mathrm{rpm}$ to $3000 \mathrm{rpm}$. The direct stiffness coefficient of the non-nominal bearing in unloaded direction $\left(k_{x x}\right)$ is always larger than (about 50\%) that of in the nominal bearing. On the other hand, the stiffness coefficients in the orthogonal loaded direction $\left(k_{y y}\right)$ show an opposite trend. These results are consistent with the level of orthotropy expected for a LOP configuration, as presented in [13].

(a)
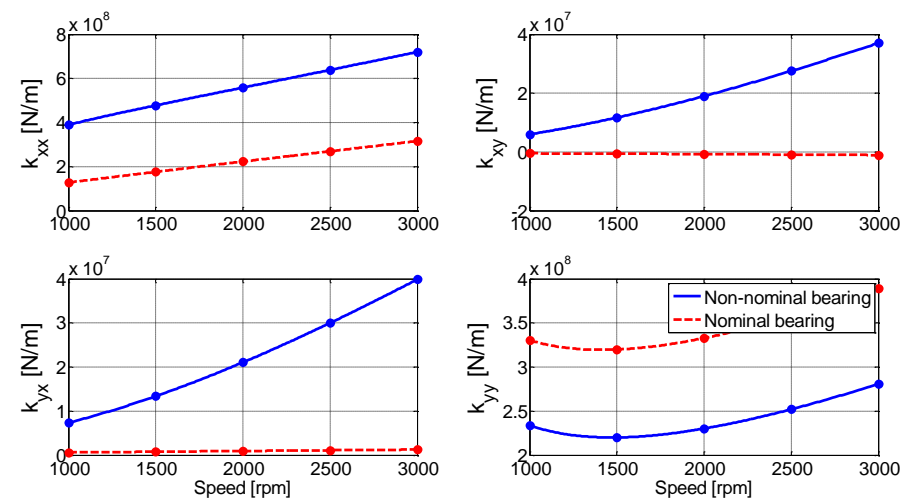

(b)
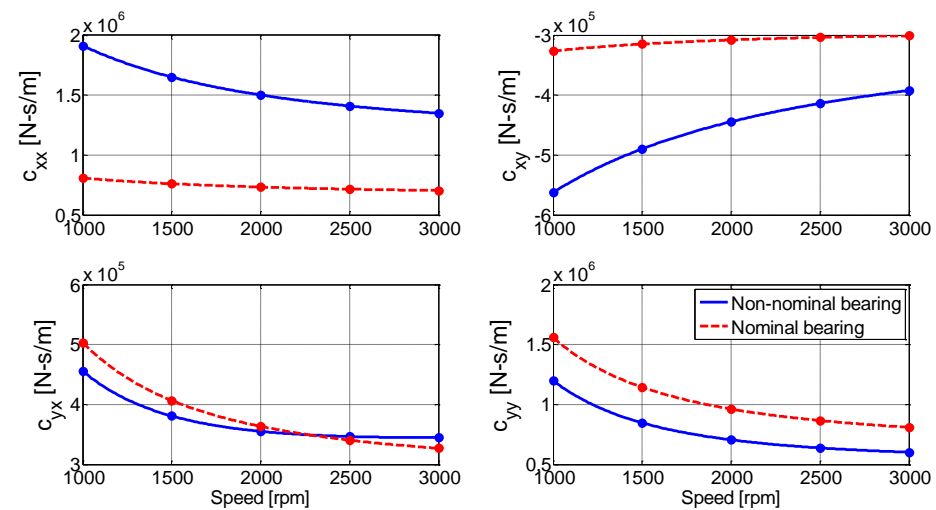

Fig. 3. Dynamic coefficients vs. rotor rotational speed

The non-nominal dimension has strong impact on cross-coupled stiffness coefficients $\left(k_{x y}\right.$ and $\left.k_{y x}\right)$. While the $k_{x y}$ and $k_{y x}$ of the nominal bearing are almost zero with rotational speed, these coefficients of the non-nominal bearing strongly depend on rotor speed, increase linearly with increasing of rotational speed. It could be explained that despite the applied load is vertical; the center locus of the non-nominal bearing is not vertical (see Fig. 4). Hence, there is a cross-coupling effect, so it is necessary to take into account also the cross-coupling dynamic coefficients $k_{x y}$ and $k_{y x}$ when the dynamic characteristics of the non-nominal bearing are evaluated.

Contrary results are shown for the direct dynamic damping coefficients. They decrease with increasing of rotational speed. For the bearing with non-nominal dimension, the direct damping in loaded direction $c_{y y}$ is less than in case of the nominal bearing, nearly about $10 \%$. 

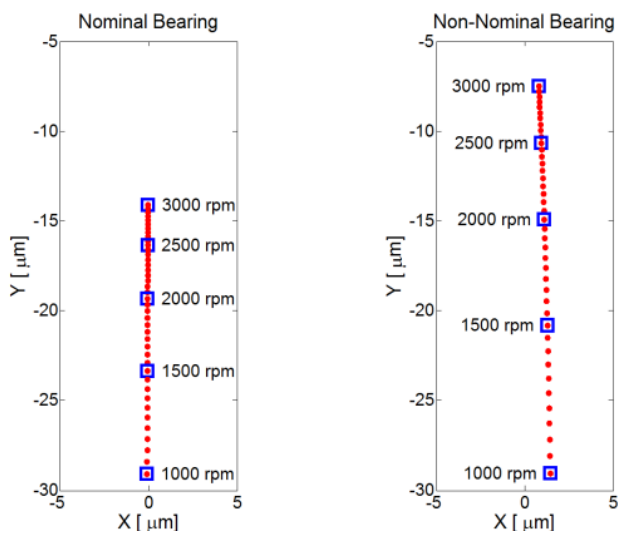

Fig. 4. Predicted shaft loci vs. rotor rotational speed

\subsection{Dynamic coefficients versus static load}

Fig. 5 shows the effect of increasing static load on the dynamic stiffness and damping coefficients for rotor speed of $1200 \mathrm{rpm}(20 \mathrm{~Hz})$ with LOP configuration.

(a)
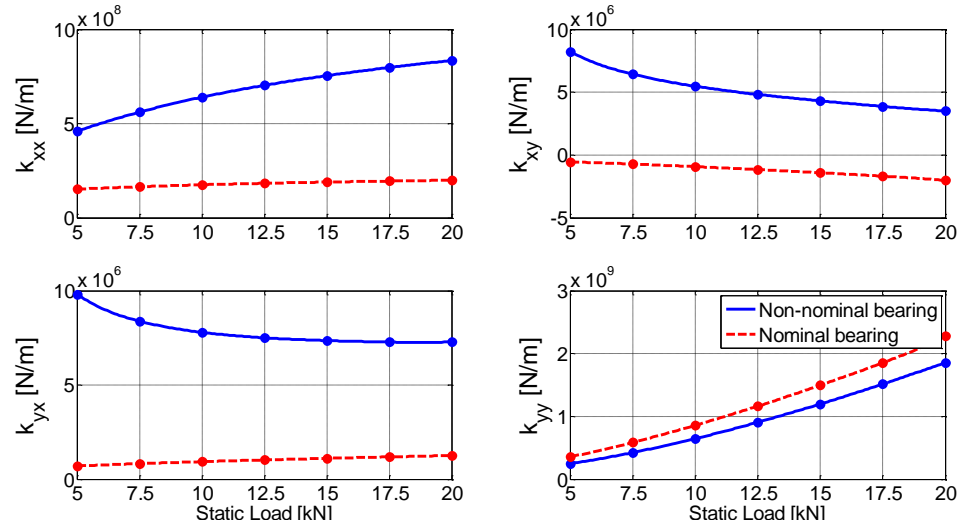

(b)
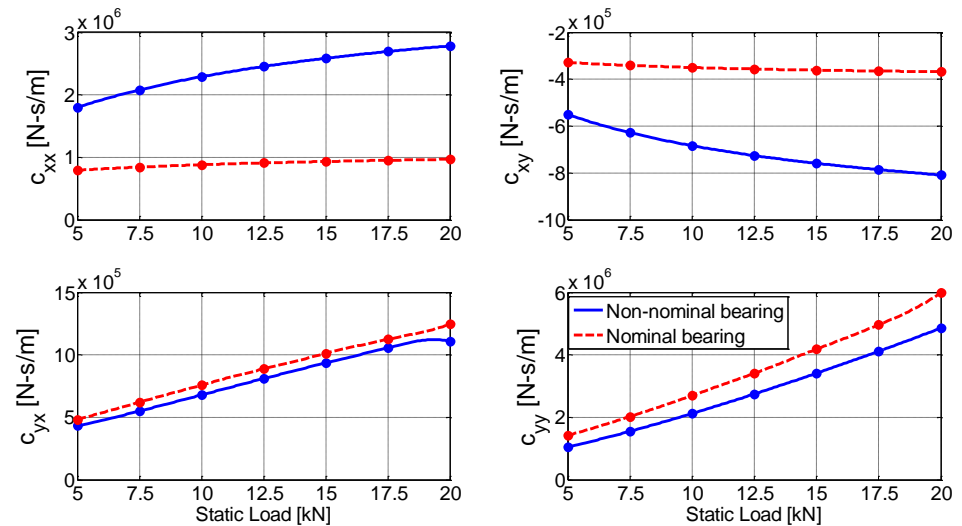

Fig. 5. Dynamic coefficients vs. static load 
While it should be noted that the direct coefficients in the unloaded direction $\left(k_{x x}\right)$ of the nominal bearing is quite stable with static load, this coefficient in the nonnominal bearing increases linearly with increasing of static load, from $4.6 \times 10^{8} \mathrm{~N} / \mathrm{m}$ at $5 \mathrm{kN}$ to $8.35 \times 10^{8} \mathrm{~N} / \mathrm{m}$ at $20 \mathrm{kN}$.

On the other hand, the dynamic direct stiffness coefficients in the loaded direction $\left(k_{y y}\right)$ on both kinds of bearing strongly depend on the static load and the stiffness coefficient of the nominal bearing is always greater (about 10\%) than that of the nonnominal one. This coefficient increases significantly, more than $600 \%$, from $3.57 \times 10^{8}$ $\mathrm{N} / \mathrm{m}$ to $2.28 \times 10^{9} \mathrm{~N} / \mathrm{m}$ for the nominal bearing and from $2.48 \times 10^{8} \mathrm{~N} / \mathrm{m}$ to $1.86 \times 10^{9}$ $\mathrm{N} / \mathrm{m}$ for the non-nominal bearing corresponding static load from $5 \mathrm{kN}$ to $20 \mathrm{kN}$. It can be seen that the cross-coupled stiffness coefficients of non-nominal bearing $\left(k_{x y}\right.$ and $k_{y x}$ ) are much larger than those of the nominal one. However these coefficients are very small in comparison with the direct coefficients on both bearings.

The similar results are shown for the dynamic damping coefficients as shown in Fig. 5 except for the cross-coupled damping coefficients $\left(c_{x y}\right.$ and $\left.c_{y x}\right)$ in which the cross coefficients of the nominal bearing are always higher than those of the nonnominal one.

\subsection{Dynamic coefficients versus load direction}

Generally, a TPJB is loaded symmetrically, i.e. load-on-pad or load-between-pad configuration, the former being the more common situation. However in practical the bearing may be loaded in any direction, not just in these two special directions. For instance, high bearing loads out of the vertical direction may occur in industrial rotating machines, like in turbo-generators, owing to bad alignment conditions of the shaft-line or in gearboxes. For the operating conditions that load direction change rapidly, it needs to perform the analysis of the bearing in its special load forms to enhance the precision and efficiency of bearing design.

Two reference systems are introduced in this section to evaluate dynamic coefficients, namely the absolute reference and the load reference systems. For the first one, a varying load direction is considered, as shown in Fig. 6. In this case, the static load is rotated in a full revolution $\left(360^{\circ}\right)$, with steps of $18^{\circ}$. It means that, all the five pads will be loaded under LOP and LBP configuration.

Fig. 7 shows the influence of the load direction on the calculated stiffness and damping coefficients of the nominal and non-nominal bearing. Obviously, the load direction has a strong effect on both of bearings' dynamic coefficients.
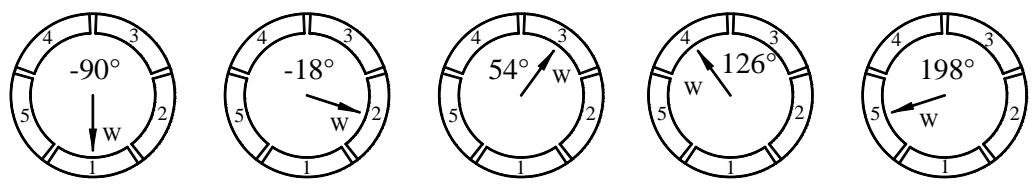

Fig. 6. Varying load configuration

The dynamic stiffness coefficients of the nominal bearing show a general good agreement with the calculated values of the non-nominal one apart from the direct 
term in horizontal direction $\left(k_{x x}\right)$. The $k_{x x}$ of the non-nominal bearing is always larger than that of the nominal one, especially when the static load is applied in the vertical (load direction is $-90^{\circ}$ ) and pad 1 is loaded. At $-90^{\circ}$, the direct stiffness coefficient in the horizontal direction $k_{x x}$ of the non-nominal bearing is about $4.3 \times 10^{8} \mathrm{~N} / \mathrm{m}$ while this value for the nominal one is nearly $1.5 \times 10^{8} \mathrm{~N} / \mathrm{m}$. The effects of load directions on the damping coefficients (Fig. 7b) are very similar to those on the stiffness terms.

(a)
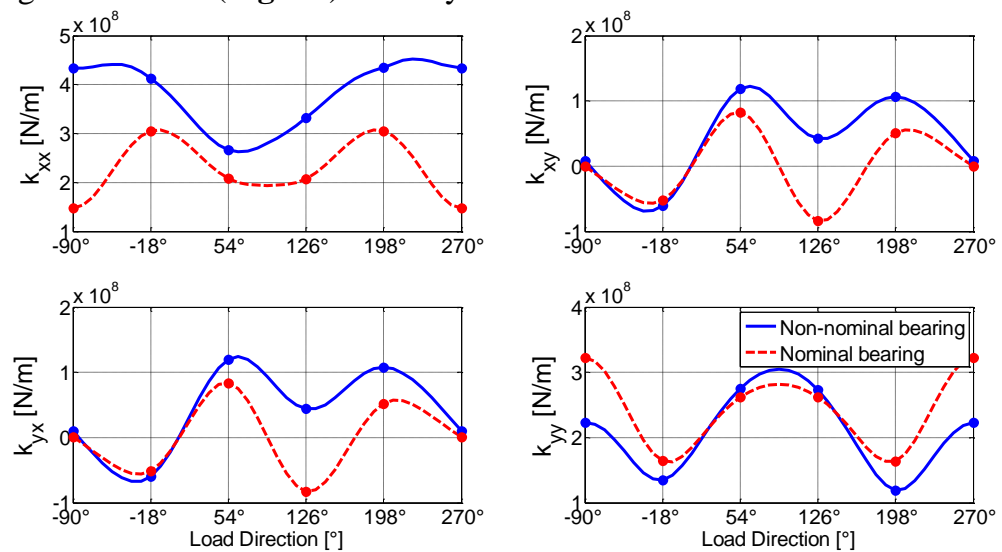

(b)
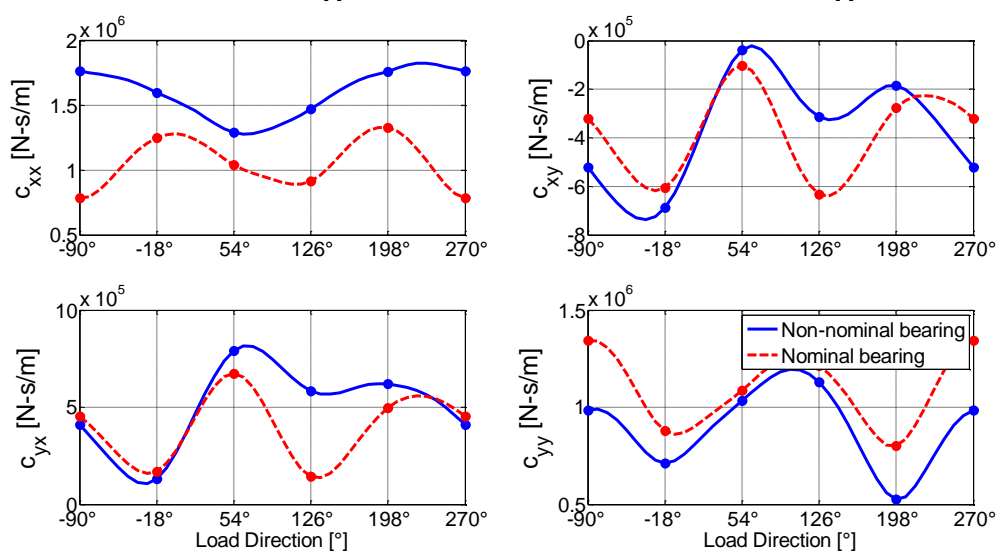

Fig. 7. Dynamic coefficients vs. load directions in absolute reference system

In the case of load reference system, the load direction is kept fixed in the vertical direction and the bearing is rotated, from $-90^{\circ}$ to $270^{\circ}$, with steps of $18^{\circ}$ with respect to the load direction, as shown in Fig. 8.
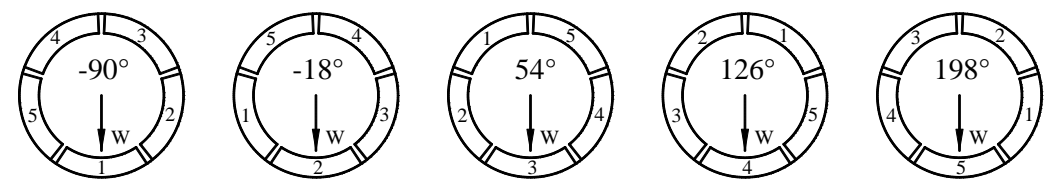

Fig. 8. Varying load configuration

The influence of load directions on the dynamic coefficients in the load reference is shown in Fig. 9. 
(a)
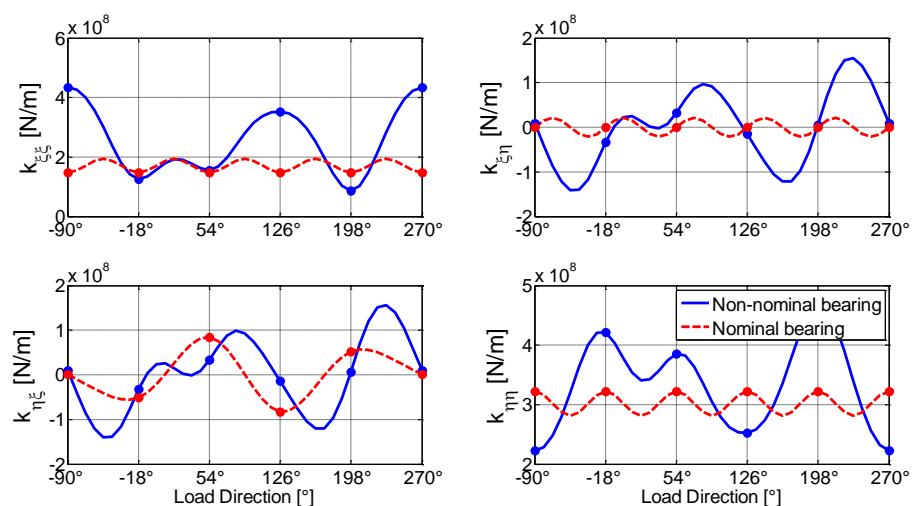

(b)
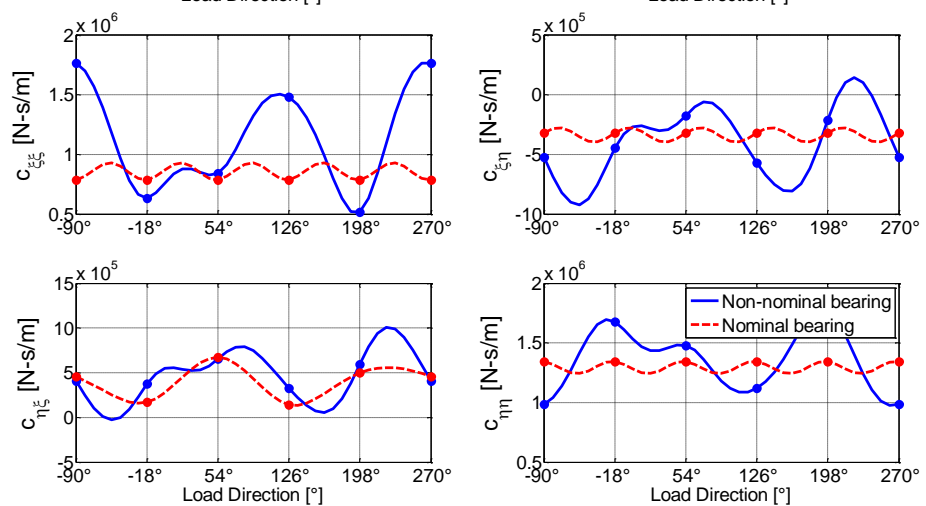

Fig. 9. Dynamic coefficients vs. load directions in load reference system

It also possible to observe that the calculated dynamic coefficients of the nominal bearing maintain a certain symmetry as a function of the direction of the load with a small variation when the static load rotates. This is less evident for the non-nominal bearing, due to asymmetric geometry. The dynamic coefficients of the non-nominal bearing show a large fluctuation with varying load directions. Similar to the previous varying load configuration, also in this case, direct term of dynamic stiffness and damping coefficients in unloaded direction $\left(k_{\xi \xi}\right.$ and $c_{\xi \xi}$ ) show the worse agreement between the nominal and the non-nominal one, among the dynamic coefficients.

\section{Conclusions}

This paper presents the dynamic characteristics of a five-pads TPJB, which is characterized by not constant assembled clearance for all the pads distribution, due to pad machining and assembling. A comparison is performed with a bearing having nominal (i.e. ideal) geometry. The following conclusions can be drawn:

1. It is necessary to take into account the cross-coupling dynamic coefficients $k_{x y}$ and $k_{y x}$ when the dynamic characteristics of non-ideal TPJB are evaluated.

2. The effect of static load on direct dynamic coefficients in the loaded direction $\left(k_{y y}\right.$ and $\left.c_{y y}\right)$ is stronger than those calculated in the unloaded direction $\left(k_{x x}\right.$ and $\left.c_{x x}\right)$. 
3. The reference system can be considered in absolute reference or in load reference when evaluating effect of load directions.

4. The load direction has a strong effect on the characteristics of TPJBs. This influence can be increased or decreased by a non-nominal geometry of the bearing, for which big differences can be identified between the experimental performance of a real bearing and the predicted behavior obtained using a model of a nominal bearing.

\section{References}

1. Lund, J.W.:Spring and Damping Coefficients for Tilting Pad Journal Bearing. In ASLE Trans., 7, 342-352 (1964).

2. Zheng, T.S, Hasebe, N.: Calculation of Equilibrium Position and Dynamic Coefficients of a Journal Bearing Using Free Boundary Theory. In Trans. ASME, J. Tribol., 122, 616-621 (2000).

3. Feng, K., Liu, W., Zhang, Z., Zhang, T.: Theoretical model of flexure pivot tilting pad gas bearings with metal mesh dampers in parallel. In Tribology International, 94, 26-38 (2016).

4. Cha, M., Glavatskih, S.: Nonlinear dynamic behaviour of vertical and horizontal rotors in compliant liner tilting pad journal bearings: some design considerations. In Tribology International, 82, 142-152 (2015).

5. Dang, P.V., Chatterton, S., Pennacchi, P., Vania, A.: Effect of the load direction on nonnominal five-pad tilting-pad journal bearings. In Tribology International, 98, 197-211 (2016).

6. Dang, P.V., Chatterton, S., Pennacchi, P., Vania, A., Cangioli, F.: Behavior of a TiltingPad Journal Bearing With Different Load Directions, ASME Paper No. DETC2015-46598, 2015; 8. doi:10.1115/DETC2015-46598.

7. Dang, P.V., Chatterton, S., Pennacchi, P., Vania, A.: Numerical investigation of the effect of manufacturing errors in pads on the behaviour of tilting-pad journal bearings. In Proceedings of the Institution of Mechanical Engineers, Part J: Journal of Engineering Tribology, 233(4), 480-500 (2018).

8. Dang, P.V., Chatterton, S., Pennacchi, P., Vania, A., Cangioli, F.: Behavior of tilting-pad journal bearings with large machining error on pads. In ASME Turbo Expo, Paper No. GT GT2016-56674 (2016).

9. Chatterton, S., Dang, P.V., Pennacchi, P, Luca, A.D., Flumian, F.: Experimental evidence of a two-axial groove hydrodynamic journal bearing under severe operation conditions. In Tribology International, 109, 416-427 (2017).

10. Chatterton, S., Dang, P.V., Pennacchi, P., Vania, A.: A test rig for evaluating tilting-pad journal bearing characteristics. In 9th International Conference on Rotor Dynamics IFToMM ICORD, Milan, Italy, 921-930 (2014).

11. Jones, G. J., and Martin, F. A.: Geometry Effects in Tilting-Pad Journal Bearings. In The 33rd Annual Meeting in Dearborn, Michigan (1978).

12. Strzelecki, S.: Dynamic characteristics of tilting 5-pads journal bearing with asymmetric support of pads. In Sixth International Conference on Rotor Dynamics (IFToMM), University of New South Wales, Sydney, Australia, 807-814 (2002).

13. Delgado, A., Vannini, G., Ertas, B. M., Drexel, M., and Naldi, L.: Identification and Prediction of Force Coefficients in a Five-Pad and Four-Pad Tilting Pad Bearing for Loadon-Pad and Load-Between-Pad Configurations. In J. Eng. Gas Turbines Power, 133(9). 